\title{
Cultural Perspective from Middle Eastern Countries on the Ethics of Involving Children with Cancer in Research
}

\section{Arabiat $\mathbf{D H}^{\star}$}

Associate professor, Department of Maternal and Child Health Nursing, Faculty of Nursing, University of Jordan, Amman, Jordan

*Corresponding author: Arabiat DH, Associate Professor, Department of Maternal and Child Health Nursing, Faculty of Nursing, University of Jordan, Amman, 11942 Jordan, Tel: +962 65355000/23182; Fax: (962777)438538; E-mail: d.arabiat@ju.edu.jo, dnarabiat@yahoo.co.uk

Received date: January 15, 2014; Accepted date: February 19 2014; Published date: February 25, 2014

Copyright: () 2014 Arabiat DH. This is an open-access article distributed under the terms of the Creative Commons Attribution License, which permits unrestricted use, distribution, and reproduction in any medium, provided the original author and source are credited.

\begin{abstract}
This paper is concerned with ethical issues that have to be considered when conducting research with children in nonwestern cultures. It sets the discussion in the context of current debate about researching children with cancer and explores the extent to which these ethical issues are similar to, or different from Western countries. A number of culturally sensitive issues encountered with children in Jordan while investigating their psychological wellbeing is discussed. These issues are discussed with relation to obtaining informed consent from children for participation in the research; issues of assuring confidentiality; and assuring no harm. Concern with children's rights has been extended to the question is it ethical to provide full information about the study for children who were not informed about their cancer diagnosis. Such issues are often overlooked and for future studies with children acknowledging these issues can help provide a basis for reasoning and direct actions. The ethical issues that are discussed in this paper exemplify that exploring children's psychological distress in a culture where this is not recognized provides the researcher with a number of concerns; the communication approaches of illness diagnosis, the wisdom of their elders, and the importance of their family which may result in reluctance to show emotion or to question decisions made on their behalf. The necessity for researchers to take an ethical stance in such cases may encounter difficulty and a number of issues had to be addressed and sorted case by case.
\end{abstract}

\section{Introduction}

The last decade has been a period of important controversies in research ethics. One of the most recent controversies includes concerns related to research with children [1]. Such concerns of the ethics of conducting research with children was a common reason for not conducting research with children; mainly related to ethical concerns over children's vulnerability to exploitation by researchers [2]. The need for informed consent, assuring of no harm and confidentiality were identified through literature as important principles that can help guide the ethical development and evaluation of clinical studies by investigators, Institutional Review Board (IRB) members, funders, and others. If these principles are fulfilled with other ethical claims, such as respect for autonomy, nonmaleficence, beneficence and justice, no further ethical requirements are needed to qualify research as ethical [3]. However, while these principles are claimed to be universally valid for all countries and contexts, they might require an adaptation to different contexts and cultures [1].

In this context, we aim to presents culturally sensitive issues encountered while investigating psychological wellbeing of children with cancer in Jordan. Although in this study we describe general cultural themes related with Jordan's society, the readers should keep in mind the variations that exist between and within national groups of Jordan's society. The partition of Palestine in 1948 and the creation of the state of Israel prompted large-scale migration of homeless Palestinian families to Jordan as refugees and they form $60 \%$ of the total population. Then, the 1991 Gulf War, which provoked conflict among the Arab countries, created another wave of Palestinian and Iraqi refugees from Kuwait and Iraq. This was followed by another wave of Syrian refugees in the last two years which is expected to add further variation into the population structure.
In terms of the social structure, the basic element of the Arab society is the family with respect to elders and suppression of personal interests for the good of the family. There are two parts dealing with child's role in the Middle East as they affect child status: the Arab culture and the Islamic faith. Therefore, in order to further understand the specific issues which have been raised in this paper, it is imperative first to illuminate the situation of children in the society. A review of studies available today reveals that little attention has been paid to the status or to the role of children in Middle Eastern society. Articles examining contextual influences based on child development in this area failed to distinguish between what is based on folk customs and what is derived from religion customs [4]. The social indicators reveal that while the religious principles of Islam accept children as persons and companion [5], the patriarchal family structure justify unquestioned authority of the father or the eldest of the tribe. This gives rise to respect to elders and suppression of personal interests for the good of the family. Lipson and Meleis [6] reported that children in the Middle East live with their parents until they marry and maintain close contact with their parents after marriage, yet the roles are reversed when parents age; the children are expected to care for their parents as long as they live.

In the process of conduction research with children in the Middle East, the need for informed consent, assuring of no harm and confidentiality are often troubled by mutual misunderstanding of culturally influenced values and communication styles. Therefore, in this study issues related with (1) obtaining informed consent for participation in the research; (2) assuring confidentiality; and (3) assuring no harm will be discussed. Concern with children's rights has been extended to the question is it ethical to provide full information 
about a research study for children who were not informed about their cancer diagnosis.

The ethical issues presented in this paper were encountered while investigating psychological wellbeing of children receiving cancer treatment at the hospitals and the schools of Jordan. Three assessment tools were translated into Arabic, piloted and tested for reliability and validity at numbers of school all around Jordan. Then, these tools were administered to children diagnosed with cancer and other chronic illness at four hospitals. Participating children were asked to complete self-reported depression (CDI); The Children Depression Inventory [7-8] and anxiety (RCMAS); The Revised Manifest Anxiety Scale $[9,10]$ scales, as well as an instrument to measure the quality of life $\left(\right.$ PedsQL $\left.{ }^{\mathrm{m}}\right)$; The Paediatric Quality of Life Inventory [11-13]. The ages of children ranged from seven years to sixteen years old. Children were invited to participate in this study regardless of their nationality or country of origin. The ethical dilemmas that were encountered are discussed in the context of children's rights and the ethical claims of research.

\section{Informed Consent in Research and Voluntary Participation}

For most researchers and IRB members informed consent is what makes research involving human subject ethical [3]. Informed consent stands on three aspects: firstly gaining knowledge through provision of full information; secondly voluntary participation and finally capacity or competency to give consent [14]. Each of the three elements is necessary to ensure that individuals make rational and free determinations of whether the research trial is consonant with their interests.

The following cases present some of the ethical dilemmas that researchers might face when conducting research with children in Middle Eastern countries. Emanuel et al. [3] argued that to provide informed consent, individuals must be accurately informed of the purpose, methods, risks, benefits, and alternatives to the research. Thus it is assumed that before children elicit their agreement they should be given honest information about the study, and this information should be commensurate with their level of intellectual and emotional maturity [15]. However, although parent and children signed consent to participate in this study, information giving presented a challenge. A number of children did not know they had cancer as their families thought it was culturally inappropriate for them to be told [16]. Thus it can be argued how it was possible to present the study for children while recruiting them, without alerting them to the fact that they had cancer.

This is a particular issue for health care professionals including nurses and researchers as a child with cancer is a vulnerable child. They are vulnerable related to their weakness, lack of knowledge and dependency, as well as to their total lack of political and civil right [17]. Given the sensitivity of the problem related with children vulnerability and of whether children knew or not their medical diagnosed the research investigator must exercise extreme caution to avoid revealing information that would identify any child as having been diagnosed or receiving cancer treatment. It is important to acknowledge here that no child was approached in this study before the parents were first asked if the child knew his/her cancer diagnosis. Each child was then asked what brought him/her to the hospital and what he/she complaining of as to assess how he/she perceived his/her illness. This gave a direction of how to ask children about the impact of their illness on their psychological wellbeing. The information sheet and the informed consent were all written without a direct referral to "impact of cancer" instead it was replaced with the "impact of illness" on their wellbeing.

In the other hand, some of the issues presented in the following case are the same as in other types of research and have their basis in moral and ethical principles. In this case, it is well documented that in the child research field permission to ask the "suicide item" of the CDI [7-8] have received attention, largely because it is not uncommon for studies using the CDI [7-8] to drop the item pertaining to suicidal ideation, for example in Shochet et al. [18]; Hannon et al. [19] and Weiss et al. [20] studies. In this study, most of the parents refused permission to ask this question to their child (65.5\% of cancer group, $\mathrm{n}=58$ and $53.6 \%$ of chronic illness group, $\mathrm{n}=56$ ), as they feared it would put ideas of death into their child's mind. Although some children got annoyed or laughed when they were asked the suicide item of the CDI [7-8] "I will kill myself" it can be argue that this is a very inappropriate item to ask for children with cancer, who may in some cases, view their illness as a death sentence.

A number of issues arise related to addressing the elements of informed consent in the previous cases: (1) How to provide full information about the study for children who were not informed about their cancer diagnosis. (2) How to balance between participants' right to be informed and potential harm that could result when children know they might have cancer. (3) If a research concerns sensitive topics is full disclosure about the selection criteria can be considered unethical? (4) After parent gives consent regarding a child's participation in research, can they act as a gatekeepers for certain questions and deny their child from answering certain questions? Indeed these are difficult questions which might have no straight answers or simple solutions. In some cases the principles might apparently conflict with each other, thus ethical evaluation of research protocols requires discussion of the ethical aspects for which principles are supposed to provide a substantive orientation [1].

\section{Anonymity and Confidentiality}

Confidentiality involves protection of the subjects' identities through anonymous responses. Confidentiality can be seen as a problematic issue in family research and school research as well where researchers are interviewing a child with the teacher presence. Obtaining a separate space away from the child's parent or the teacher can be a sensitive issue in the Arab world, as some adults may not see children as having a need for privacy. For example, during the piloting of the RCMAS [9] at one of the primary schools of Jordan, the presence of the social worker while children were filling in the questionnaires in the classroom raised a problem (the social workers referred to at schools are in fact teachers who have taken on a more pastoral role in the school and not what may be considered social workers in the western countries). The teacher's general comments to motivate the children, "You are from nice good families", "You've got to answer well and help the researcher to get her results" indeed were made with the good intention of obtaining perfect answers. However, the question arises whether these comments invaded the children's right to privacy and independent answers? Might the questions themselves lead to problems in the child if he felt responsible for the research outcome? If such comments are made to children, will it lead to a sense of guilt about the way they felt about themselves or their relations with their parents and environment? 
Another element of the research situation requiring special consideration concerns test answers revealing emotional problems in the child. The question for the investigator is whether to inform the child's parents or any third party about such negative findings and offer referrals for help. Moreover, if the child has been promised that his/her answers will not be revealed to anyone then can the investigator inform the parents of the child's potential problems without breaching the confidentiality promised the child. [15] argued that if the consent agreement signed by parents and children promises confidentiality of data, then the investigator cannot inform any third party or make any direct referrals for assistance without violating the assurance of confidentiality. Although there are researchers who treat all information as confidential, most experts consider that complete confidentiality cannot be guaranteed and that researchers have a duty to pass on information if they feel a child is 'at risk' [14]. Decisions concerning whether to inform parents or others about an individual child's answers must balance the parents' right to know against the child's right to privacy [15].

One of the situations encountered in the current study and which require special attention occurred when three children's answers for the suicide item of the CDI [7-8] and some spontaneous comments lead the investigator to suspect clinical depression and risk for suicide. The ethical dilemma faced was whether or not to report the result and related risk. The question was if parents or school and hospital social workers have the right to be given information about things children have answered in confidence? Doesn't this violate the confidentiality promised for both parents and child? Will failing to report this risk and making referrals increase the risk of harm to the child? Does confidentiality with treating information regarding suicidal ideation cannot be guaranteed in this case?

Previous studies highlighted the limits to confidentiality in information sheets, especially when disclosing information that suggests that child or other lives may be at risk [14]. Issues related with limits to confidentiality in information sheets should be highlighted in each research as well as how the researchers will manage disclosure $[2,14,21]$. In the developing countries, the issue of referring children in risk to third parties is complicated by the availability of professional help. Given that researchers may not be able to refer children in need to professionals, there remains the question of whether it is moral or ethical for the investigator to leave these children coping with their negative feelings alone. With the evident lack of funds for intervening studies, those children are not likely to receive counseling in any case.

It is important to acknowledge that the children's test result on the CDI [7-8] in the previous case was reported to the hospital social worker. The response which was received after referring these children was not to take the children's responses seriously, as they were affected by the news media and they wanted to present themselves as heroes and not afraid of death. The social worker response has been instrumental in raising numerous ethical issues and controversies. These issues provoked a longstanding -discussion about many facets of research including the degree to which such responses among children about the "suicide item" of the CDI [7-8] in the Arab world are universal to depression symptoms or culture specific to the Middle Eastern countries. Numerous studies document facing issues with using the suicide item of the CDI [7-8] among children and have shown that children's responses were a reaction to the protective approach of families. In this case, Jordan is no exception; as a culture, it is widely known that families avoid any discussion of related topics of death, it is not uncommon to send children away to a relative's or a friend's house when some close person dies or during funerals.

Though it seems attempting to link the suicide item with culturespecific issues linked with "martyrdom" concept in the middle eastern countries, it is unwise to make cultural assumptions using such comments without further validation of those responses and comments. While the risks involved in failing to report suspected suicide risk for a depressed child in this case are not negligible, the risks resulting from incongruous referral are far more serious. The potential harm resulting from underestimation of children's emotional wellbeing may adversely contribute to biased and ignorant attitudes based on misconceptions of a community who might lack education and understanding.

Furthermore, the appropriateness of providing information about child's performance has been another issue of some debate. Hutz and Koller [22] dispute that if a child's answers or tests scores are not shared with parents, should investigators provide parents with general information about their child's result? Moreover, what information should be given to the children themselves regarding their performance? In the current study, issues of providing information about the child's test result seemed very important for some of the children that most children interviewed at schools would not usually think of filling in a questionnaire without being told how the questionnaire was scored. The fact that scoring instructions were not provided with the questionnaires presented difficulty, as some of the students view test scores as a meaningful exam, and expected to pass or to fail. To avoid the impact of being referred to as having given right or wrong answers, additional explanation of the study was given to the students at each school. Nevertheless, a significant percentage of returned questionnaires had names on them, and most students sought individual feedback about their answers. Although it might be assumed that children will feel more confident to answer anonymously, we should bear in mind that in the Arab world some children participants interpreted the anonymous responses as lack of affection, and as being treated like inanimate objects, which could lead to their feeling vulnerable, powerless and deserted. On the other hand, wouldn't children feel less free to answer questions honestly if they know that others may identify their answers?

\section{No Harm}

Researchers have an ethical responsibility to ensure that children do not suffer harm from participating in research [21]. This is of great concern in the child research literature because children are vulnerable and incompetent. Kirk [2] argued that children taking part in research may feel distress, conflict, guilt, threat to self-esteem, fear of failure and embarrassment. Such concern was experienced during validating the anxiety tool into Arabic language at one of Jordan's school. A twelve-year old female student burst into tears; she started complaining of headache and pain in her knees after completing the RCMAS [9]. The same pattern of communication was repeated with another female student where no medical problem was a matter of concern for either of them. The dilemma for the researcher concerns was what steps to take beyond referring those children to the social worker. The observed explanation for the above case has been established beyond a shadow of doubt as somatization. It is well documented in literature that anxiety or depression symptoms may be expressed differently in different cultures; in particular some in nonewestern cultures may somatise their symptoms of distress [23-25]. In this instance, should the investigator inform the child's parents of the 
child's somatisation? Would the parental knowledge of the child's somatisation may adversely affect the parent-child relationship. Mudaly and Goddard [26] argue that while serious ethical difficulties arise regarding how to balance the welfare rights of children to be protected from harm with their right to be consulted, strategies which empower minimise risks are possible. It is therefore important to be sensitive to local cultural issues where children may show reluctant to express their true negative emotion and to be ready to contend with any similar emotions experienced as a result of child participating. Regardless of whether children show any evidence of stress, investigators should probe to determine what aspects of the research are upsetting to the child and be able to clarify any misperception and ensure that the child do not suffer any negative consequences from participation in the research [15].

\section{Conclusion}

The literature about the ethics and practice of research with children assent that informed consent; no harm and confidentiality are important principles in any research. This means that each ethical principle has to be fulfilled with the exception of informed consent in special cases [1]. Cancer research, however, generates specific ethical problems in developing countries where some children might not know their cancer diagnosis. Investigating psychological wellbeing of children here is especially difficult because of their vulnerability and lack of power in the clinical situation. Therefore the potential conflict between child's right and parent's protective approach has to be solved. The few cases presented here shows that there are no clear-cut or black and white answers when defining children's right in research. Hence while undertaking studies with children; researchers had to address a number of difficult ethical and methodological issues. The first is our need to consider the cultural and methodological issues that have been debated in the social sciences and critically reflect on the practicability of involving children as active agents in non-Western communities. A second issue is the vulnerability of children and their need for protection from potential exploitation by researchers. Researchers also need to pay attention to the significant role of family in the Arab world. Underestimation of emotional wellbeing of children may adversely contribute to biased and ignorant attitudes based on misconceptions of a community who might lack education and understanding. Nurses who attempt to conduct research with children have to consider a variety of complex ethical issues, which are addressed in this paper. Ethical situations are common and diverse in research as well as it varies among different cultures. Although situations can vary, the ethical principles remain universal. As indicated earlier, there are no clear solutions to these dilemmas or white and black answers. The process of moral reflection and cultural sensitivity will help researchers to justify their actions.

The issues that have been described here are derived primarily from clinical and research experience. Though the need for informed consent, assuring of no harm and confidentiality are often troubled by mutual misunderstanding of culturally influenced values and communication styles, they do share a core of universal needs and common values. Despite of any difficulties encountered, children in this society are emotionally expressive that the researcher gets immediate feedback and high response rate. The issues discussed here are common guidelines that hope to provide an understanding of the cultural characteristics of this society and, therefore, will improve rather than impede conducting further studies with this group. A personal approach and affiliation to family by the researcher in this society can help to bridge the gap and gives the enriching opportunity to experience the child's world and the significance of family of Middle Eastern society.

\section{References}

1. Ehni HJ (2010) Elaborating and promoting a new ethical framework for clinical research Med Health Care and Philos 13: 287-290.

2. Kirk S (2007) Methodological and ethical issues in conducting qualitative research with children and young people: A literature review. Int J Nurs Stud 44: 1250-1260.

3. Emanuel EJ, Wendler D, Grady C (2000) What Makes Clinical Research Ethical? JAMA, 283: 2701-2711.

4. Arabiat D (2013) Psychological Wellbeing of Children with Cancer in Jordan. LAP LAMBERT Academic Publishing.

5. Hedayat KM, Pirzadeh R (2001) Issues in Islamic Biomedical Ethics: A Primer for the Pediatrician. Pediatrics. 108: 965-971

6. Lipson JG, Meleis Al (1983) Issues in health care of Middle Eastern patients, In Cross cultural medicine. West J Med 139: 854-86.

7. Kovacs M (1981) Rating scales to assess depression in school-aged children. Acta Paediopsychiatrica, 46: 305-315.

8. Kovacs M (1992) Children's Depression Inventory Manual. Los Angeles: Western Psychological Services.

9. Reynolds CR, Ricmond BO (1985) Revised Children's Manifest Anxiety Scale (RCMAS) Manual. Los Angeles: Western psychological Service.

10. Al Jabery M, Arabiat D (2011) Psychometric properties of the Arabic translated version of the RCMAS, preliminary indicators from a Jordanian sample. Journal of International Counselor Education 3:13-24

11. Varni JW, Burwinkle TM, Seid M, Skaree D (2003) The PedsQL $L^{\mathrm{mm}} 4.0$ as a pediatric population health measure; feasibility, reliability, and validity. Ambul Pediatr 3: 329-341.

12. Varni JW, Seid M, Rode CA (1999) The PedsQL ${ }^{m}$ : measurement model for the pediatric quality of life inventory. Med Care 37: 126-139.

13. Arabiat D, Elliott B, Draper P, Al Jabery M (2011) Cross-cultural Validation of the Pediatric Quality of Life Inventory ${ }^{\mathrm{mm}} 4.0$ (PedsQL $^{\mathrm{nt}}$ ) generic core scale into Arabic language. Scand J Caring Sci 25: 828-833

14. Beresford B (1997) Personal Accounts: Involving Disabled Children in Research. Social Policy Research Unit, New York.

15. Kinard EM (1985) Ethical Issues in Research with Abused Children, Child Abuse Negl 9: 301-311.

16. Arabiat DH, Alkaisi NM Hamdan-Mansour A (2011) Children's knowledge of their cancer diagnosis and treatment: Jordanian mothers' perceptions and satisfaction with the process. Int Nurs Rev 58: 443-449

17. Morrow V, Richard M (1996) The Ethics of Social Research with Children: An Overview, Children \&Society 10: 90-105.

18. Shochet IM, Dadds MR, Holland D, Whitefield K, Harnett PH, Osgarby S (2001) The efficacy of a universal school-based program to prevent adolescent depression. J Clin Child Psychol 30: 303-315.

19. Hannon AP, Rapee RM, Hudson JL (2000) The prevention of depression in children: A pilot study. Behaviour Change 17: 78-83.

20. Weiss B, Weisz JR, Politano M, Carey M, Nelson WM, et al. (1991) Developmental differences in the factor structure of the Children's Depression Inventory. Psychological Assessment: A Journal of Consulting and Clinical Psychology 3: 38-45.

21. Alderson P (1995) Listening to the children; children, ethics and social research. Barkingside, UK: Branardos.

22. Hutz CS, Koller SH (1999) Methodological and Ethical Issues in Research with Street Children. New Dire Child Adolesc Dev 85: 59-70.

23. Zhang D (1995) Depression and culture: A Chinese perspective. Canadian Journal of Counseling 29: 227-223.

24. Al-Lawati J, Al-Lawati N, Al-Siddiqui M, Antony SX, Al-Naanani A, et al. (2000) Psychological morbidity in primary healthcare in Oman: a preliminary study. SQU Journal for Scientific Research Medical Sciences 2: 87-90. 
Citation: Arabiat DH (2014) Cultural Perspective from Middle Eastern Countries on the Ethics of Involving Children with Cancer in Research. Clinics Mother Child Health 11: 159. doi:10.4172/2090-7214.1000159

Page 5 of 5

25. Tobin JP (2000) Observation on the mental health of civilian population living under-term hostilities. Psychiatric Bulletin 24: 69-70.
26. Mudaly N (2009) The Ethics of Involving Children Who Have Been Abused in Child Abuse Research. The International Journal of Children's Rights 17: 261-281. 